\title{
Optical Tracking of Anomalous Diffusion Kinetics in Polymer Microspheres
}

\author{
Matthew R. Foreman* and Frank Vollmer \\ Max Planck Institute for the Science of Light, Laboratory of Nanophotonics and Biosensing, \\ Günther-Scharowsky-Straße 1, 91058 Erlangen, Germany
}

(Received 15 December 2014; revised manuscript received 29 January 2015; published 17 March 2015)

\begin{abstract}
In this Letter we propose the use of whispering gallery mode resonance tracking as a label-free optical means to monitor diffusion kinetics in glassy polymer microspheres. Approximate solutions to the governing diffusion equations are derived for the case of slow relaxation and small Stefan number. Transduction of physical changes in the polymer, including formation of a rubbery layer, swelling, and dissolution, into detectable resonance shifts are described using a perturbative approach. Concrete examples of poly(methyl methacrylate) and polystyrene spheres in water are considered.
\end{abstract}

DOI: 10.1103/PhysRevLett.114.118001

PACS numbers: 81.05.Lg, 07.07.Df, 42.60.Da, 66.30.jj

Diffusion in polymers is a complex process, the understanding of which can bring great benefit to, for example, design of polymer membranes, microfluidics, fuel cells, controlled drug delivery systems, and sensors [1-7]. Fundamentally, many physical principles can influence the diffusion kinetics, such that differing diffusion regimes have been observed ranging from so-called Fickian (case I) diffusion, in which the rate of penetrant diffusion dominates the kinetics, through anomalous diffusion, to non-Fickian (case II) diffusion governed by polymer relaxation [8,9]. Consequently, both theoretical modeling $[10,11]$ and development of associated experimental techniques, such as Fourier transform infrared attenuated total reflection, nuclear magnetic resonance spectroscopy, fluorescence imaging, laser interferometry, and gravimetry [12-16], have seen long-standing research interest. Comprehensive understanding and control of diffusion kinetics, however, still remains a challenge, such that further means by which to monitor the diffusion process precisely are still of great importance.

In this work we propose a novel label-free method of monitoring diffusion in glassy polymers, e.g., polyethylene terephthalate (PET), based on tracking of whispering gallery mode (WGM) resonances in a polymer microsphere. While the high $Q$ factors of these surface-type modes provide a sensitive method of studying diffusion kinetics or sensing of volatiles, this work also provides a, hitherto lacking, quantitative description of the degradation and swelling processes affecting polymer biosensors, which are invariably used in aqueous environments [5,7]. We primarily concern ourselves with polymer-penetrant systems suffering relatively small physical changes from penetrant diffusion, as may result from, for example, low penetrant solubility, slow dissolution rates, and small molar volume. Environmental plastic pollutants represent an important class of such systems, the study of which can profit from the high sensitivity afforded by WGM tracking. We begin this Letter by introducing the diffusion model used, which serves to demonstrate the pertinent effects of diffusion on WGMs. We derive approximate asymptotic solutions that further allow physical insights before presenting the electromagnetic model describing transduction of physical changes in the polymer into optically detectable signals. Perturbative results for induced resonance shifts are also given. Distinct stages in the diffusion kinetics are finally identified and discussed.

When a dry glassy polymer microsphere is immersed into a solvent [17], a complex mixture of diffusion, polymer swelling, and erosion can occur (see Fig. 1). Initially, as the solvent begins to diffuse into the outermost regions of a dry polymer, a phase transition is induced whereby glassy polymer transforms into a rubbery state as a result of plasticization and polymer relaxation [8]. Over time the boundary between the glassy and rubbery regions advances towards the center of the sphere. Similarities with a classical one-phase Stefan problem are manifest [18]; however, the polymer network in the rubbery layer simultaneously expands so as to accommodate solute molecules, resulting in a swelling of the sphere. Inclusion of the second moving boundary, therefore, requires extension of the Stefan solution. Once polymer entanglement becomes suitably small, the sphere can ultimately undergo surface dissolution such that the outer surface begins to retreat and the microsphere shrinks [19].

To model these various aspects of solvent diffusion, we extend the models of Refs. [20-22]. We begin by adopting Fick's law of diffusion whereby

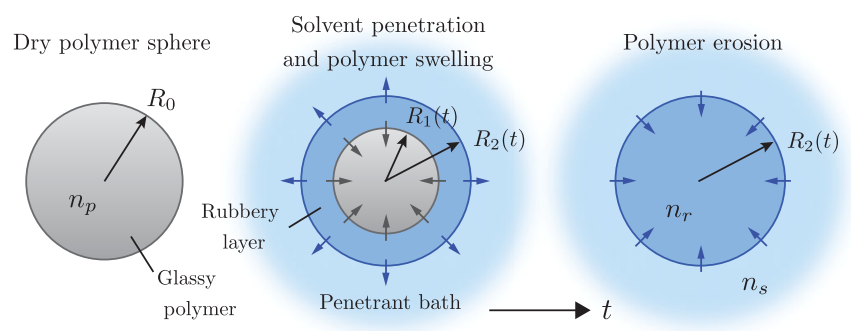

FIG. 1 (color online). Schematic of penetrant dynamics upon introduction of a glassy polymer microsphere into a solvent bath. Arrows depict direction of movement of the polymer interfaces. 


$$
\frac{\partial U}{\partial t}=\frac{\mathcal{D}}{R^{2}} \frac{\partial}{\partial R}\left(R^{2} \frac{\partial U}{\partial R}\right),
$$

where $U=U(R, t)$ is the penetrant molar concentration, $R$ and $t$ are the radial and time coordinates, and $\mathcal{D}=D\left[D_{g}\right]$ for $R_{1}(t) \leq R \leq R_{2}(t)\left[0 \leq R<R_{1}(t)\right]$ is the diffusion coefficient of the penetrant in the rubbery [glassy] region. Note that $R_{1}(0)=R_{2}(0)=R_{0}$. Since the glass-rubber interface is generally sharp, the phase transition is modeled as a threshold process occurring at a penetrant concentration of $U^{*}$ [8]. Assuming that the polymer sphere is immersed in a penetrant bath, we can assert $U=U_{0}>U^{*}$ for $R=R_{2}(t)$, where $U_{0}$ is the solubility of the penetrant in the polymer; i.e., a condition of local equilibrium holds. We further assume that the velocity of the glass-rubber interface depends on the excess penetrant concentration at the interface above the threshold value $[23,24]$, i.e.,

$$
\frac{d R_{1}}{d t}=-K\left(U-U^{*}\right)^{\gamma} \text { at } R=R_{1}(t),
$$

where the kinetic parameter $K$ and nonlinear coefficient $\gamma$ are phenomenological constants. Introduction of the kinetic parameter $K$ regularizes unphysical singularities in the classical one-phase Stefan problem arising when the glassrubber interface reaches the center of the sphere [25]. For simplicity, we henceforth assume $\gamma=1$. Nonlinear dynamics have been investigated in Ref. [25].

Mass conservation at the inner interface implies

$$
\left.D_{g} \frac{\partial U}{\partial R}\right|_{R_{1}-\delta}-\left.D \frac{\partial U}{\partial R}\right|_{R_{1}+\delta}=\left[U\left(R_{1}, t\right)-U^{*}\right] \frac{d R_{1}}{d t},
$$

with $\delta \rightarrow 0$ [26]. Typically, $D_{g} \ll D[9,27]$ such that the so-called Fickian precursor penetrating into the glassy region exhibits a rapid falloff with a decay length $L\left(D_{g}\right)$. Documented values of $D_{g}$ are, however, rare. In lieu of adopting an arbitrary value of $D_{g}$, we instead make an adiabatic approximation [11] allowing diffusion in the glassy and rubbery regions to be decoupled. When $L \ll R_{0}$, the solvent concentration in the core can be approximated as $U \approx U^{*} \exp \left[-\left(R-R_{1}\right) D_{g}^{-1} d R_{1} / d t\right]$ [11], which can then be substituted into Eq. (3) to eliminate $D_{g}$ [28]. In contrast, when $L \gtrsim R_{0}$, size-dependent diffusion kinetics can result [39] and the assumption of a sharp glass-rubber interface no longer holds since the penetrant concentration quickly reaches $U^{*}$ throughout the core. Therefore, we assume $L \ll R_{0}$; however, note that this restriction poses no difficulty since WGMs are also supported in larger (e.g., macroscopic) spheres. Errors introduced through use of an approximate solution are largest when $R_{1}(t) \lesssim L$, which proves unimportant in our WGM tracking scheme (see below).

Penetrant mass that diffuses into the polymer has a finite volume and hence causes the sphere to expand. The penetrant flux across the outer boundary follows from modifying Fick's first law to account for the moving boundary viz. $J=4 \pi R_{2}^{2}\left(D \partial U / \partial R+U_{0} d R_{2} / d t\right)$. Further considering surface erosion, the total rate of change of the volume $V$ is given by $d V / d t=\bar{\nu} J-4 \pi R_{2}^{2} K_{s}$, where $\bar{\nu}$ is the penetrant molar volume and $K_{s}$ is the surface normalized erosion coefficient. Hence, we arrive at the final boundary condition

$$
\frac{d R_{2}}{d t}=\bar{\nu} D \frac{\partial U}{\partial R}+\bar{\nu} U_{0} \frac{d R_{2}}{d t}-K_{s} \quad \text { at } \quad R=R_{2}(t) .
$$

No analytic solution to Eqs. (1)-(4) is known and hence they must be solved numerically. With appropriate scaling [28] and introduction of the parameters $\mu=D\left(U_{0}-U^{*}\right)^{-\gamma} /$ $\left(K R_{0}\right)$ and $\sigma=U^{*} /\left(U_{0}-U^{*}\right)$, known as the nondimensional kinetic parameter and Stefan number, respectively, Eqs. (1)-(4) can be nondimensionalized [28] to facilitate such computations. Importantly, it should be noted that after a time $t=t_{f}$, the glass-rubber interface reaches the center of the sphere. Instead of Eqs. (2) and (3), a zero flux condition must thus be enforced at $R=0$ for $t \geq t_{f}$ [28].

To illustrate the behavior of the above equations, we consider a poly(methyl methacrylate) (PMMA) and a polystyrene (PS) microsphere of $50 \mu \mathrm{m}$ radius immersed in water. Anomalous diffusion behavior has been reported in both systems [40,41]. Dissolution rates are negligible in these systems; however, for simulation purposes, it is necessary to input the associated values of $D, U^{*}, U_{0}$, and $K$ (see Table I). Threshold concentrations were determined using the Fujita-Kishimoto method [42] whereby the threshold mass fraction is given by $C^{*}=\alpha_{f}\left(T_{g}-T\right) / \beta$, where $T_{g}(T)$ is the glass transition (ambient) temperature of the polymer, $\beta$ is a characteristic polymer-penetrant parameter, and $\alpha_{f}$ is the polymer free-volume constant [28]. Values of $K$ were estimated using published experimental values of the penetrant front velocity or saturation time $[43,44]$. Numerical solution of the nondimensionalized differential equations was performed by applying a front-fixing transformation before subsequent use of the method of lines and standard finite difference techniques [22,26,28]. Small time asymptotic solutions, valid for $\tau=O\left(\mu^{2}\right)$, where $\tau=D t / R_{0}^{2}$ is the scaled time, were used to seed the solution [28]. Such asymptotic solutions also allow us to approximately express the condition $L \ll R_{0}$ as $\mu D_{g} / D \ll 1$. Figure 2 shows the resulting interface positions as a function of time (solid lines) for both the

TABLE I. Simulation parameters (see also Ref. [28]).

\begin{tabular}{lcc}
\hline \hline & PMMA-water & PS-water \\
\hline$D\left(\mathrm{~cm}^{2} \mathrm{~s}^{-1}\right)$ & $3.35 \times 10^{-8}$ & $1.7 \times 10^{-7}$ \\
$U_{0}\left(\mathrm{~mol} \mathrm{~m}^{-3}\right)$ & 986.392 & 466.079 \\
$U^{*}\left(\mathrm{~mol} \mathrm{~m}^{-3}\right)$ & 115.320 & 107.815 \\
$K\left(\mathrm{~m}^{4} \mathrm{~s}^{-1} \mathrm{~mol}^{-1}\right)$ & $2.646 \times 10^{-10}$ & $1.597 \times 10^{-11}$ \\
Stefan number $\sigma$ & 0.1324 & 0.3009 \\
Nondimensional kinetic parameter $\mu$ & 0.2906 & 59.4204 \\
\hline \hline
\end{tabular}




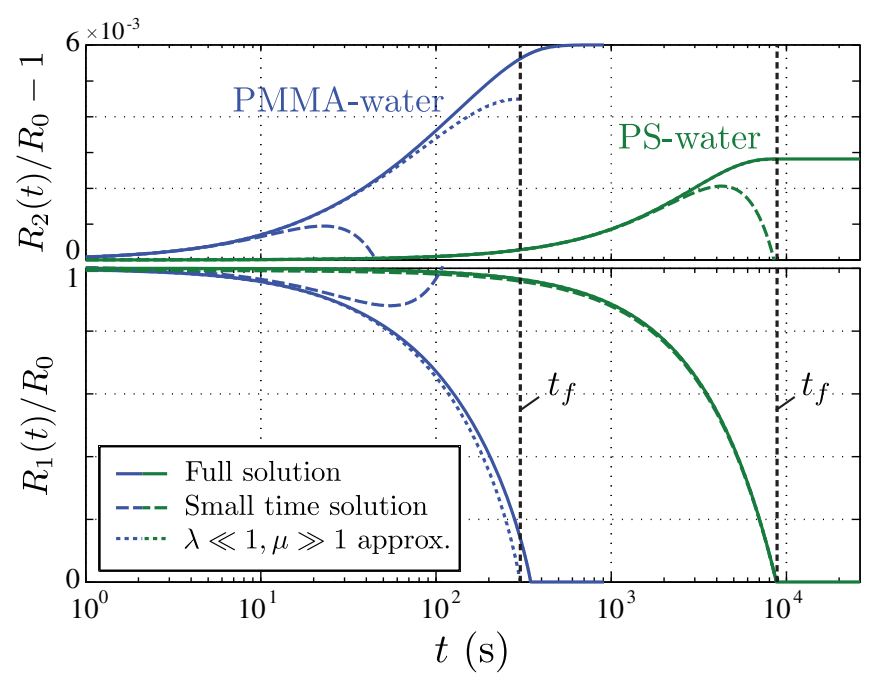

FIG. 2 (color online). Temporal evolution of microsphere surface (top) and glass-rubber interface (bottom) for PMMA-water (blue lines) and PS-water (green lines) polymer-penetrant systems.

PMMA- and PS-water system, along with the small time asymptotic solutions (dashed lines). A number of points of interest are apparent. Firstly, the higher solubility of water in PMMA gives rise to a larger swelling of PMMA microspheres. The total fractional change in $R_{2}$, however, represents only a fraction of a percent, or equivalently $\lambda / 4$, assuming an optical wavelength of $\lambda \approx 1210 \mathrm{~nm}$. Swelling of such a small magnitude can nevertheless transduce large resonance shifts as will be seen below. Secondly, we note that the entire diffusion process is slower for the PS system, despite a higher diffusion coefficient. Specifically, saturation of the PMMA (PS) microsphere takes 5 (146) min. Primarily, this behavior results from the slower rate at which PS relaxes, as reflected by the larger value of $\mu$. Under the conditions $\sigma \ll 1$ and $\mu \gg 1$, it is possible to find approximate expressions for the evolution of the interfaces. Derivation is given in the Supplemental Material [28], whence we find

$$
\begin{aligned}
\tau= & \left\{\left(5-4 \rho_{1}\right) \mu+\left(1-2 \rho_{1}\right) M_{1}+(1+\mu) \operatorname{arccot} \sqrt{\mu}\right. \\
& \left.+(1+\mu) \arctan \left[\left(1-2 \rho_{1}\right) M_{1}^{-1} \sqrt{\mu}\right]\right\} / 8, \\
& \rho_{2}^{3}=1+\frac{6 \nu}{\nu-1} \int_{0}^{\tau} \frac{\rho_{1}^{2}}{\mu+2 \rho_{1}-2 \rho_{1}^{2}+M_{1}} d \tau^{\prime},
\end{aligned}
$$

where $M_{1}=\left[\mu^{2}+4 \mu\left(\rho_{1}-\rho_{1}^{2}\right)\right]^{1 / 2}$ and $\rho_{j}=\rho_{j}(\tau)=R_{j}(t) / R_{0}$ for $j=1,2$. These solutions are shown in Fig. 2 by the dotted lines. Near perfect agreement is seen for the PS case. Discrepancies for the PMMA microsphere occur since $\mu=O(1)$ and are largest at times $\tau \sim \tau_{f}$. Specifically, a diminished swelling and faster advance of the glass-rubber interface are predicted, the former of which is most significant in determining shifts of WGM resonances (see below). Noting that saturation occurs when $R_{1} \approx 0$, the saturation time follows from Eq. (5) as $\tau_{f} \approx[3 \mu+\sqrt{\mu}(1+\mu) \operatorname{arccot} \sqrt{\mu}] / 4$, in good agreement with numerical results. We further note the resulting $R_{0}^{2}$ scaling of $t_{f}$.

Whispering gallery modes supported in polymer microspheres are highly sensitive to the local environment and the resonator radius $R_{2}$. For homogeneous microspheres, the resonance frequency $\omega$ can be found using the asymptotic approximation [45]

$$
n \omega R_{2} / c \approx v+2^{-1 / 3} \zeta_{q} v^{1 / 3}+O(1),
$$

where $l$ is the WGM order, $v=l+1 / 2, c$ is the speed of light, $q$ is the radial order, $\zeta_{q}$ denotes the $q$ th zero of the Airy function $\operatorname{Ai}(-z)$, and $n$ is the refractive index of the microsphere. Diffusion of solvent into the microsphere, however, produces a radial inhomogeneity in the refractive index profile, thus invalidating Eq. (7). For simplicity, we make an effective medium approximation, such that the refractive index of the rubbery layer is $n_{r}=\eta_{s} n_{s}+\eta_{p} n_{p}$, where $\eta_{s}$ and $\eta_{p}$ are the volume fraction of the solvent (of refractive index $n_{s}$ ) and polymer $\left(n_{p}\right)$, respectively. An effective refractive index accounting for the Fickian precursor can be used in place of $n_{p}$; however, given that the difference is small, we neglect this effect. Accordingly, resonant frequencies can be found via numerical root finding of the associated resonance condition [46]. To help reduce the associated numerical complexity, we also present a perturbative approach. Formulas exist in the literature for thin layers of arbitrary refractive index difference [47]; however, here we develop expressions valid for layers of arbitrary thickness with small refractive index differences. Derivation is again given in the Supplemental Material [28]; however, we find that the shift in resonance frequency, upon introduction of a layer of refractive index $n_{r}$, from that of a homogeneous sphere of the same outer radius and with refractive index $n_{p}$, is

$$
\frac{\delta \omega}{\omega} \approx \frac{\epsilon_{0} l(l+1)}{4 U_{l m}^{\nu}}\left(n_{p}^{2}-n_{r}^{2}\right) \Omega^{\nu} \mathcal{I}_{3}^{\nu},
$$

where $\nu=$ TE or TM denotes the polarization of the WGM, $\Omega^{\mathrm{TE}}=\omega^{2} \mu_{0}^{2}, \Omega^{\mathrm{TM}}=1, \epsilon_{0}\left(\mu_{0}\right)$ is the permittivity (permeability) of free space, and $U_{l m}^{\nu}$ are given in Ref. [48]. For TE modes, we have $\mathcal{I}_{3}^{\mathrm{TE}}=\mathcal{F}_{3}^{0}\left(R_{2}\right)-\mathcal{F}_{3}^{0}\left(R_{1}\right)$, where

$$
\mathcal{F}_{3}^{0}(x)=x^{2} \frac{j_{l}\left(z_{p}\right) j_{l}\left(z_{r}\right)}{\left(n_{p}^{2}-n_{r}^{2}\right) k}\left[n_{p} \frac{j_{l+1}\left(z_{p}\right)}{j_{l}\left(z_{p}\right)}-n_{r} \frac{j_{l+1}\left(z_{r}\right)}{j_{l}\left(z_{r}\right)}\right] .
$$

$k=2 \pi / \lambda, z_{j}=n_{j} k x$, and $j_{l}(z)$ denotes the spherical Bessel function. See the Supplemental Material [28] for the TM case [28]. Figure 3 shows the evolution of TE polarized WGMs of differing radial order in a PS microsphere, initially at $\lambda \approx 1210 \mathrm{~nm}$, found using Eq. (8) and numerical root finding, whereby good agreement is seen. Physically, three distinct regimes can be identified (shown for $q=1$ ), as determined by the thickness of the rubbery layer $T$, relative to the radial extent $\Delta R_{l q} / R_{2} \approx$ $(3 \pi)^{2 / 3} / 2\left[(q-1 / 4) /\left(n_{p} k R_{0}\right)\right]^{2 / 3}$ of the WGM [45]. 


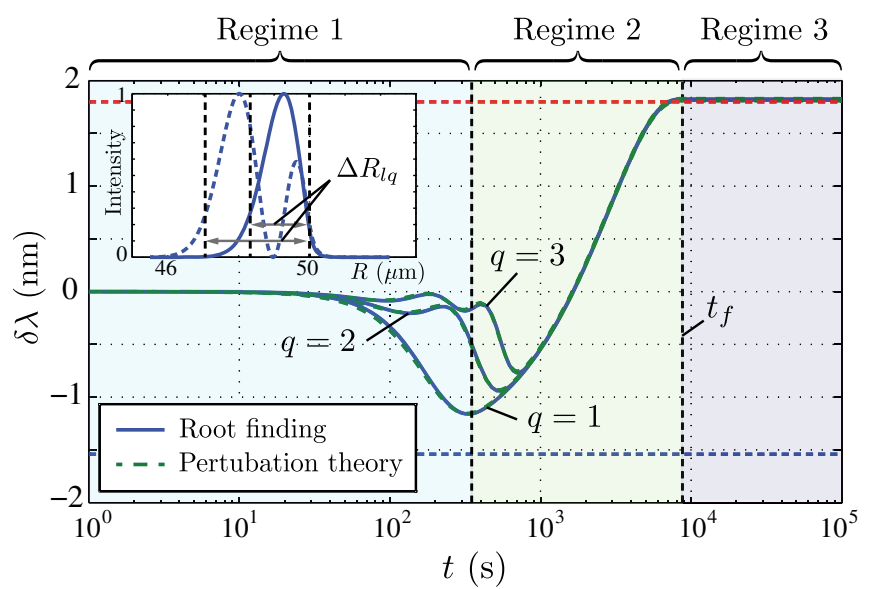

FIG. 3 (color online). Temporal evolution of resonance wavelength shift $\delta \lambda$ for PS-water system for $(l, q)=(396,1),(386,2)$, and $(377,3)$ fundamental WGMs. Blue and red dashed lines depict the predictions of Eqs. (11) and (12), respectively, for $q=1$. Inset: Radial extent of WGMs in a $50 \mu \mathrm{m}$ PS microsphere.

In each case, differing processes govern the observed resonance shift, as we now discuss in turn.

Regime 1: Effective refractive index phase $T \lesssim \Delta R_{l q}$. Initially, the diffusing penetrant is limited to a thin layer near the resonator surface and the glass-rubber interface moves across the mode volume, thereby inducing a change in the refractive index $\Delta n$ seen by the WGM. Simultaneously, the polymer sphere swells by $\Delta R$ such that the total resonance shift results from both effects. The relative importance of each can be estimated by considering a rubbery layer of thickness $\Delta R_{l q}$ and volume $V_{l}$. In this scenario, we can use Eq. (7) to estimate the net shift as $\Delta \omega / \omega \approx-\Delta n / n_{p}-\Delta R / R_{0}$. We consider the limiting case of a saturated layer. Accordingly, the volume of solvent in the layer is given by $V_{s}=V_{l} \bar{\nu} U_{0}$. Noting then that the associated volume fractions of solvent (polymer) are $\eta_{s}=$ $\bar{\nu} U_{0}\left(\eta_{p}=1-\bar{\nu} U_{0}\right)$, we find $\Delta n \approx \bar{\nu} U_{0}\left(n_{s}-n_{p}\right)$. Given $n_{p}>n_{s}$, an increase in resonant frequency, i.e., a blueshift, results. Solvent ingress, however, also causes an increase in the microsphere volume of $V_{s}$, such that $\Delta R \approx \bar{\nu} U_{0} \Delta R_{l q}$, hence inducing a redshift of the WGM. The relative magnitude of the opposing shifts is thus approximately

$$
\left|\frac{\left.\Delta \omega\right|_{\Delta n=0}}{\left.\Delta \omega\right|_{\Delta R=0}}\right| \approx \frac{(3 \pi)^{2 / 3} n_{p}}{2\left(n_{p}-n_{s}\right)}\left[\frac{q-1 / 4}{n_{p} k_{0} R_{0}}\right]^{2 / 3} .
$$

Given the $R_{0}^{-2 / 3}$ scaling, blueshifts evidently dominate in larger microspheres. For the PMMA and PS spheres considered here, a unity ratio corresponds to $R_{0} \sim 10$ and $5 \mu \mathrm{m}$, respectively, for a $\lambda \approx 1210 \mathrm{~nm}, q=1$ mode, such that only low $l$ lossy modes are supported. Using the constraint $n \omega R \approx$ const, we can more accurately estimate the maximum resonance shift in regime 1 as

$$
\frac{\Delta \omega_{1}}{\omega} \approx \frac{n_{p}\left(1-\bar{\nu} U_{0} \Delta R_{l q}\right)}{n_{p}+\bar{\nu} U_{0}\left(n_{s}-n_{p}\right)}-1
$$

The dashed blue line in Fig. 3 depicts the maximum shift predicted by Eq. (11). Slight overestimation arises from assumption of a saturated layer. For higher order radial modes, the wavelength shift oscillates as the glass-rubber interface advances across each successive field maxima. Maximum blueshifts are also smaller.

Regime 2: Swelling phase $T \gg \Delta R_{l q}$.-Once the rubbery layer becomes much thicker than the radial extent of the WGM, the swelling of the microsphere dominates the induced resonance shift, since the polymer within the modal volume is essentially saturated. Only redshifts are hence observed. The maximum redshift can be estimated similarly to above, yielding

$$
\frac{\Delta \omega_{2}}{\omega} \approx \frac{n_{p}\left(1-\bar{\nu} U_{0}\right)^{1 / 3}}{n_{p}+\bar{\nu} U_{0}\left(n_{s}-n_{p}\right)}-1 .
$$

Without the competing blueshifts arising from refractive index changes, the sensitivity of the WGM resonance to polymer swelling is maximum during this stage of diffusion. Observing that the minimum resolvable shift of the WGM is a small fraction $f$ (typically $\approx 0.01$ ) of the resonance linewidth [49,50], it follows that the minimum observable fractional change in the microsphere radius $\approx f Q^{-1}$. Neglecting additional absorption from the penetrant, typical absorption limited $Q$ factors of $\sim 1.7 \times 10^{5}$ are obtainable for PMMA and PS microspheres $\left(Q_{\mathrm{abs}}=n_{p} k / \alpha\right.$, where $\alpha$ is the polymer absorption coefficient $[28,51])$. In turn, this implies that for a $50 \mu \mathrm{m}$ sphere, the minimum additional penetrant volume $\Delta V_{\min }$ required to induce a resolvable resonance shift is $\sim 3 f V Q^{-1} \approx 90 \mathrm{aL}$, representing a high sensitivity for detecting solvent penetration.

Regime 3: Dissolution phase $T \approx R_{2}, \tau \gtrsim \tau_{f}$. - In the final stage of the diffusion process, the polymer becomes saturated with penetrant, such that swelling ceases. Dissolution, however, results in shrinkage of the microsphere and a blueshift of the WGM. Noting that once saturated $\partial U / \partial R=\partial n_{r} / \partial t=0$, Eqs. (4) and (7) give

$$
\frac{1}{\omega} \frac{d \omega}{d t} \approx \frac{K_{s} / R_{0}}{1-\bar{\nu} U_{0}-K_{s} t / R_{0}} .
$$

Since $K_{s} / R_{0} \ll 1$ and $\bar{\nu} U_{0} \ll 1$, it follows that $\omega(t) \approx$ $\omega_{0} K_{s} t / R_{0}$ irrespective of which mode is tracked. Briefly considering dissolution of PMMA in methyl isobutyl ketone $\left(K_{s} \sim 0.091 \mu \mathrm{m} / \mathrm{min}\right.$ at $20^{\circ} \mathrm{C}$ [52]), we note that the resonance wavelength shifts at a rate of $d \lambda / d t \approx$ $-3.34 \mathrm{pm} / \mathrm{s}$. Better solvents give rise to more rapid evolution of the WGM; however, this can become experimentally difficult to observe. Use of larger spheres can mitigate this issue. Bulk dissolution of the polymer also produces similar behavior, since in this case the refractive index of the resonator changes linearly with time, instead of the resonator size.

In summary, this Letter proposes monitoring diffusion kinetics in polymers by means of tracking WGMs in polymer microspheres upon immersion in a penetrant bath. Similar phenomena can also be exploited in, e.g., cylindrical 
resonators. Practically, we note that experimental realization requires a good control of ambient temperature so as to avoid associated resonance drifts. Initial environmental conditions must also be controlled, since ambient moisture may result in preliminary formation of a rubbery layer extending beyond the WGM mode, therefore rendering the first stage of diffusion unobservable. Nevertheless, high sensitivity real time monitoring of polymer swelling can be simply achieved since this process occurs over longer time scales and regardless of initial layer formation. Weak polymer dissolution can also be easily observed using WGM tracking, to such an extent that monitoring of slow degradation at rates on the order of $\mu \mathrm{m} / \mathrm{yr}$, as are relevant for degradation of environmental plastic pollutants, e.g., PET and PS, is conceivable. Finally, theoretically this Letter contributes new formulas describing diffusion kinetics in glassy polymers from which estimates of saturation time follow. Such formulas aid understanding and facilitate extraction of unknown material properties through fitting. Moreover, such knowledge is useful for predicting the lifetime and stabilization times of polymer sensors when used in aqueous environments.

This work was funded through an Alexander von Humboldt Fellowship (M. R. F.) and the Max Planck Society (F. V.). We also acknowledge discussions with Dr. R. Keding (Max Planck Institute for the Science of Light, Erlangen) and Dr. S. Yang (Brigham and Women's Hospital, Boston).

*matthew.foreman@mpl.mpg.de

[1] R. Tharanathan, Trends Food Sci. Tech. 14, 71 (2003).

[2] G. C. Randall and P. S. Doyle, Proc. Natl. Acad. Sci. U.S.A. 102, 10813 (2005).

[3] M. H.-N. Hsieh, S. W. McCue, T. J. Moroney, and M. I. Nelson, ANZIAM J. 52, C549 (2011).

[4] N. W. DeLuca and Y. A. Elabd, J. Power Sources 163, 386 (2006).

[5] J. Lutti, W. Langbein, and P. Borri, Appl. Phys. Lett. 93, 151103 (2008).

[6] S. Mehrabani, P. Kwong, M. Gupta, and A. M. Armani, Appl. Phys. Lett. 102, 241101 (2013).

[7] A. François, N. Riesen, H. Ji, S. Afshar V, and T. M. Monro, Appl. Phys. Lett. 106, 031104 (2015).

[8] T. Alfrey, E. F. Gurnee, and W. G. Lloyd, J. Polym. Sci. Part C 12, 249 (1966).

[9] N. L. Thomas and A. H. Windle, Polymer 23, 529 (1982).

[10] L. Masaro and X. X. Zhu, Prog. Polym. Sci. 24, 731 (1999).

[11] T. Qian and P. L. Taylor, Polymer 41, 7159 (2000).

[12] Y. A. Elabd, M. G. Baschetti, and T. A. Barbari, J. Polym. Sci. B 41, 2794 (2003).

[13] M. J. Williamson, H. V. S. A. Hubbard, and I. M. Ward, Polymer 40, 7177 (1999).

[14] T. Cherdhirankorn, A. Best, K. Koynov, K. Peneva, K. Muellen, and G. Fytas, J. Phys. Chem. B 113, 3355 (2009).

[15] A. Y. Abramov, V. P. Ryabukho, and A. B. Shipovskaya, Tech. Phys. 52, 1576 (2007).

[16] J. Crank and G. S. Park, Diffusion in Polymers (Academic Press, London, 1968).
[17] The terminology solvent and penetrant will be used interchangeably in this work regardless of solubilities.

[18] H. Hu and S. A. Argyropoulos, Model. Simul. Mater. Sci. Eng. 4, 371 (1996).

[19] B. A. Miller-Chou and J. L. Koenig, Prog. Polym. Sci. 28, 1223 (2003).

[20] D. S. Cohen and T. Erneux, SIAM J. Appl. Math. 48, 1466 (1988).

[21] J.-S. Lin and Y.-L. Peng, Int. J. Heat Mass Transfer 48, 1186 (2005).

[22] M. H.-N. Hsieh, Ph.D. thesis, Queensland University of Technology, 2012.

[23] G. Astarita and S. Joshi, J. Membr. Sci. 4, 165 (1978).

[24] D. S. Cohen and T. Erneux, SIAM J. Appl. Math. 48, 1451 (1988).

[25] S. W. McCue, M. H.-N. Hsieh, T. J. Moroney, and M. I. Nelson, SIAM J. Appl. Math. 71, 2287 (2011).

[26] T. C. Illingworth and I. O. Golosnoy, J. Comput. Phys. 209, 207 (2005).

[27] P. I. Lee, Polymer 34, 2397 (1993).

[28] See Supplemental Material at http://link.aps.org/ supplemental/10.1103/PhysRevLett.114.118001, which contains Refs. [29-38], for further derivations.

[29] M. Abramowitz and I. A. Stegun, Handbook of Mathematical Functions (Dover Publications, New York, 1972).

[30] D. S. Eisenberg and W. Kauzmann, The Structure and Properties of Water (Oxford University Press, Oxford, 2005).

[31] J. E. Mark, Polymer Data Handbook (Oxford University Press, Oxford, 1999).

[32] G. M. Hale and M. R. Querry, Appl. Opt. 12, 555 (1973).

[33] N. Sultanova, S. Kasarova, and I. Nikolov, Acta Phys. Pol. 116, 585 (2009).

[34] R. C. Progelhof, J. Franey, and T. W. Haas, J. Appl. Polym. Sci. 15, 1803 (1971).

[35] M. Karimi, in Mass Transfer in Chemical Engineering Processes, edited by J. Markos (InTech, Rijeka, 2011).

[36] D. T. Turner, Polymer 28, 293 (1987).

[37] L. M. Folan, Appl. Opt. 31, 2066 (1992).

[38] B. Narasimhan and N. A. Peppas, J. Pharm. Sci. 86, 297 (1997).

[39] J. X. Li and P. I. Lee, Polymer 47, 7726 (2006).

[40] G. A. Pogany, Polymer 17, 690 (1976).

[41] D. T. Turner, Polymer 23, 197 (1982).

[42] N. A. Peppas and N. M. Franson, J. Polym. Sci., Part B: Polym. Phys. 21, 983 (1983).

[43] C. S. Brazel and N. A. Peppas, Biomaterials 20, 721 (1999).

[44] J. Reiclin, E. Bormashenko, A. Sheshnev, and R. Pogreb, Proc SPIE 4129, 305 (2000).

[45] S. Schiller, Appl. Opt. 32, 2181 (1993).

[46] M. R. Foreman and F. Vollmer, New J. Phys. 15, 083006 (2013).

[47] I. Teraoka and S. Arnold, J. Opt. Soc. Am. B 23, 1381 (2006).

[48] M. R. Foreman, S. Avino, R. Zullo, H.-P. Loock, F. Vollmer, and G. Gagliardi, Eur. Phys. J. Spec. Top. 223, 1971 (2014).

[49] M. R. Foreman, W.-L. Jin, and F. Vollmer, Opt. Express 22, 5491 (2014).

[50] L. Shao, X.-F. Jiang, X.-C. Yu, B.-B. Li, W. R. Clements, F. Vollmer, W. Wang, Y.-F. Xiao, and Q. Gong, Adv. Mater. 25, 5616 (2013).

[51] M. L. Gorodetsky, A. A. Savchenkov, and V. S. Ilchenko, Opt. Lett. 21, 453 (1996).

[52] R. J. Groele and F. Rodriguez, "Dissolution Rates of Polymers and Copolymers Based on Methyl, Ethyl, and Butyl Methacrylate", Defense Technical Information Center, Cornell University Ithaca, School of Chemical Engineering, 1988. 